\title{
Remote Postconditioning Alone and Combined with Hypothermia Improved Postresuscitation Cardiac and Neurological Outcomes in Swine
}

\author{
Jiefeng Xu, ${ }^{1,2}$ Zeng Huang, ${ }^{1,2}$ Sen Ye, ${ }^{1,2}$ Moli Wang, ${ }^{1,2}$ Ya Fang, ${ }^{1,2}$ and Zilong Li ${ }^{1,2}$ \\ ${ }^{1}$ Department of Emergency Medicine, Yuyao People's Hospital, Medical School of Ningbo University, Ningbo, China \\ ${ }^{2}$ Institute of Emergency Medicine, Medical School of Ningbo University, Ningbo, China \\ Correspondence should be addressed to Zilong Li; lizilong55@vip.sina.com
}

Received 4 September 2016; Revised 20 November 2016; Accepted 29 November 2016

Academic Editor: Hideo Inaba

Copyright (C) 2016 Jiefeng Xu et al. This is an open access article distributed under the Creative Commons Attribution License, which permits unrestricted use, distribution, and reproduction in any medium, provided the original work is properly cited.

\begin{abstract}
Objective. Previously, we demonstrated that remote ischemic postconditioning (RIpostC) improved postresuscitation myocardial and cerebral functions in rat. Here, we investigated the effects of RIpostC alone and combined with therapeutic hypothermia (TH) on cardiac and neurological outcomes after CPR in swine. Methods. Twenty-one pigs were subjected to 10 mins of VF and then 5 mins of CPR. The animals were randomized to receive RIpostC alone, or its combination with $\mathrm{TH}$, or sham control. RIpostC was induced by 4 cycles of limb ischemia followed by reperfusion. TH was implemented by surface cooling to reach a temperature of 32-34 ${ }^{\circ} \mathrm{C}$. Results. During $72 \mathrm{hrs}$ after resuscitation, lower level of cardiac troponin I and greater stroke volume and global ejection fraction were observed in animals that received RIpostC when compared to the control. RIpostC also decreased serum levels of neuron-specific enolase and $\mathrm{S} 100 \mathrm{~B}$ and increased neurologic alertness score after resuscitation. The combination of RIpostC and TH resulted in greater improvement in cardiac and neurological outcomes than $\mathrm{RIpostC}$ alone. Conclusion. RIpostC was conducive to improving postresuscitation myocardial and cerebral functions and reducing their organ injuries. Its combination with $\mathrm{TH}$ further enhanced its protective effects.
\end{abstract}

\section{Introduction}

Cardiac arrest (CA) is always a leading cause of death worldwide. It is estimated that 325,000 victims suffer out-ofhospital CA in the United States, accompanied with 350,000 in Europe and 544,000 in China every year [1-3]. Despite advances in cardiopulmonary resuscitation (CPR) methods and post-CA care, the improvement remains poor in survival rate and survival to hospital discharge with good neurological outcome in CA victims $[4,5]$.

The high rates of mortality and morbidity are mainly attributed to the severity of post-CA disease, which is typically characterized by brain damage, myocardial dysfunction, systemic inflammatory response, and coagulation disorder [6]. It is reported that $45 \%-60 \%$ of resuscitated patients exhibit severe and lethal myocardial dysfunction, which has been implicated as a major cause of early death after successful resuscitation $[7,8]$. Moreover, postresuscitation brain injury contributes to $68 \%$ of the death in CA victims, and nearly $60 \%$ survivors have moderate to severe cognitive deficits three months after resuscitation $[9,10]$.

Earlier, remote ischemic postconditioning (RIpostC) emerged as an endogenous form of organ protection and has shown its potent protection of heart and brain against regional ischemia reperfusion (IR) injury [11-14]. Recently, we applied RIpostC in a rat model of CA and resuscitation and demonstrated that RIpostC significantly attenuated postresuscitation myocardial and cerebral dysfunctions and improved the duration of survival [15]. Further investigations are necessary to confirm the effectiveness of RIpostC in larger-animal studies and clinical trials of CPR.

Currently, therapeutic hypothermia $(\mathrm{TH})$ has been introduced into the clinic as the most effective treatment to reduce neurological impairment following successful CPR [16]. Additionally, some investigations have confirmed that mild hypothermia not only alleviated postresuscitation 
neurological dysfunction, but also improved cardiac outcomes after resuscitation [17-19]. However, the improvement in postresuscitation neurological outcome and survival produced by $\mathrm{TH}$ is limited. Clinically following successful resuscitation, only $35.7 \%$ of out-of-hospital CA and $41 \%$ of in-of-hospital CA achieved a good neurological outcome at hospital discharge $[20,21]$. New strategies should be explored to produce a greater effect of organ protection after resuscitation, which may be achieved by combining $\mathrm{TH}$ and other treatments.

In the present study, we translated the previous smallanimal experiment into a clinically relevant, large-animal model. We investigated the effects of RIpostC on the outcomes of heart and brain after CPR in swine. In addition, the potential synergistic effect produced by combined RIpostC and $\mathrm{TH}$ was examined. We hypothesized that RIpostC would alleviate postresuscitation myocardial and cerebral dysfunctions and their organ injuries, which would be enhanced by its combination with $\mathrm{TH}$.

\section{Materials and Methods}

2.1. Study Design. This was a prospective, randomized, controlled experimental study. A porcine model of cardiac arrest and resuscitation was utilized. The protocol of this study was approved by the Animal Care and Use Committee of the Medical School of Ningbo University. Animal care and experiments were conducted according to Institutional Animal Care and Use Committee guidelines.

2.2. Animal Preparation. Twenty-one male domestic pigs weighing $37 \pm 2 \mathrm{~kg}$ were fasted overnight except for free access to water. Anesthesia was initiated by intramuscular injection of ketamine $(20 \mathrm{mg} / \mathrm{kg})$ and completed by an ear vein injection of sodium pentobarbital $(30 \mathrm{mg} / \mathrm{kg})$. An additional dose of sodium pentobarbital $(8 \mathrm{mg} / \mathrm{kg})$ was injected at hourly intervals to maintain anesthesia. A cuffed endotracheal tube was advanced into the trachea. The animals were mechanically ventilated with a volume controlled ventilator (SynoVent E5, Mindray, Shenzhen, China) with a tidal volume of $15 \mathrm{ml} / \mathrm{kg}$, peak flow of $40 \mathrm{l} / \mathrm{min}$, and $\mathrm{FiO}_{2}$ of 0.21 . Endtidal carbon dioxide $\left(\mathrm{ETCO}_{2}\right)$ was continuously monitored with a handheld $\mathrm{ETCO}_{2} / \mathrm{SPO}_{2}$ monitor (PMSH-300, SunLife Science Inc., Shanghai, China). Respiratory frequency was adjusted to maintain $\mathrm{ETCO}_{2}$ between 35 and $40 \mathrm{~mm} \mathrm{Hg}$. The conventional lead II electrocardiogram was continuously monitored by applying three adhesive electrodes to the shaved skin of the right upper and left upper-and-lower limbs.

For the measurement of myocardial function such as stroke volume (SV) and global ejection fraction (GEF), a PiCCO Monitor (PiCCOplus, Pulsion Medical Systems, Munich, Germany) based on transpulmonary thermodilution was applied. A $7 \mathrm{~F}$ central venous catheter was inserted into the right internal jugular vein for the injection of iced saline. Another $4 \mathrm{~F}$ thermistor-tipped arterial catheter was inserted into the left femoral artery. The arterial and central venous catheters were connected to the PiCCO system for discontinuous monitoring of SV and GEF. For the measurement of aortic pressure and the collection of blood samples, a fluid-filled $8 \mathrm{~F}$ catheter (Model 6523, C. R. Bard Inc., Salt Lake, UT) was advanced from the right femoral artery into the thoracic aorta. For the measurements of right atrial pressure and core (blood) temperature, a $7 \mathrm{~F}$ Pentalumen, thermodilution tipped catheter (Abbott Critical Care \# 41216, Chicago, IL) was advanced from the right femoral vein into the right atrium. All catheters were flushed intermittently with saline containing 5 IU bovine heparin per $\mathrm{ml}$. For inducing ventricular fibrillation (VF), a $5 \mathrm{~F}$ pacing catheter (EP Technologies Inc., Mountain View, CA) was advanced from the right external jugular vein into the right ventricle. The position of all catheters was confirmed by characteristic pressure morphology and with fluoroscopy. Blood temperature was maintained at $37.5 \pm 0.5^{\circ} \mathrm{C}$ during baseline with the aid of a cooling/warm blanket.

\subsection{Experimental Procedure}

2.3.1. Animal Randomization. Fifteen minutes prior to inducing VF, baseline measurements were obtained. The animals were randomized with the Sealed Envelope Method into one of the three groups: (1) RIpostC, (2) RIpostC+TH, or (3) sham control.

2.3.2. Induction of $V F$ and $C P R$. VF was induced by $1 \mathrm{~mA}$ alternating current through the $5 \mathrm{~F}$ pacing catheter, delivered to right ventricular endocardium. Mechanical ventilation was discontinued after onset of VF. Prior to initiating the resuscitation protocol, the pacing catheter was withdrawn to avoid heart injury during chest compression. After 10 mins of untreated VF, CPR was manually performed by a ratio of $30: 2$ of compression to ventilation. The position of chest compression was located in the midline at the level of the fifth interspace. The compression quality was continuously monitored by an E Series Monitor Defibrillator (ZOLL Medical Corporation, Chelmsford, MA) to guarantee a compression depth of $\geq 50 \mathrm{~mm}$ at a rate of $\geq 100$ per minute. The ventilation was performed by a CPR simple respirator with room air. After $2.5 \mathrm{mins}$ of $\mathrm{CPR}$, the first bolus of epinephrine at a dose of $20 \mu \mathrm{g} / \mathrm{kg}$ was administered. After 5 mins of CPR, defibrillation was attempted with a single 150$\mathrm{J}$ biphasic waveform electrical shock delivered between the conventional right infraclavicular electrode and the apical electrode with the E Series Monitor Defibrillator. If an organized rhythm with a MAP of greater than $50 \mathrm{~mm} \mathrm{Hg}$ persisted for an interval of 5 mins or more, the animal was regarded as successfully resuscitated. With failure to achieve return of spontaneous circulation (ROSC), chest compression and ventilation were immediately resumed for 2 mins prior to another defibrillation. The protocol was repeated until successful resuscitation or for a total of 15 mins. Additional doses of epinephrine were administered at an interval of 3 mins after the first bolus injection. If recurrent VF occurred after resuscitation, a 150-J electrical shock was attempted.

2.3.3. Application of RIpostC and TH. RIpostC was induced at the beginning of CPR. Four cycles of remote ischemia were performed in animals that received RIpostC. Each cycle of remote ischemia was performed by 5 mins of bilateral 
upper limb ischemia followed by reperfusion for 5 mins. Limb ischemia was induced by inflating a $5 \mathrm{~cm}$ wide blood pressure cuff around the upper third of the limb to stop the arterial blood supply. During the ischemic period, the cuff was inflated to $200 \mathrm{~mm} \mathrm{Hg}$ for 5 mins, followed by deflation for $5 \mathrm{~min}$ reperfusion. At $5 \mathrm{mins}$ after resuscitation, $\mathrm{TH}$ was implemented by surface cooling with the aid of a cooling blanket and ice packs to reach a temperature of $32-34^{\circ} \mathrm{C}$ until $4 \mathrm{hrs}$ after resuscitation, followed by a rewarming rate of $1^{\circ} \mathrm{C} / \mathrm{h}$ for 4 hrs.

2.3.4. Postresuscitation Care. Following ROSC, mechanical ventilation was continued with $\mathrm{FiO}_{2}$ of 0.21 for $4 \mathrm{hrs}$. Thereafter, all catheters were removed and wounds were surgically sutured. The endotracheal tube was removed when the animal recovered from anesthesia and had spontaneous respiration. The animals were then returned to their cages and observed for an additional $68 \mathrm{hrs}$. The surviving animals had free access to water and food but no other treatments. At the end of $72 \mathrm{hrs}$ after resuscitation, the animals were euthanized with an intravenous injection of $150 \mathrm{mg} / \mathrm{kg}$ sodium pentobarbital. A necropsy was routinely performed for documentation of possible injuries to the bony thorax and thoracic and abdominal viscera resulting from the surgical or CPR intervention or the presence of obfuscating diseases.

2.4. Measurement. Electrocardiogram, aortic and right atrial pressures, blood temperature, and pulse oxygen saturation were continuously recorded by a patient monitoring system (BeneView T6, Mindray, Shenzhen, China). Coronary perfusion pressure (CPP) was calculated as the difference between decompression diastolic aortic and time-coincident right atrial pressure measured at the end of each minute of precordial compression. The value of $\mathrm{ETCO}_{2}$ was continuously recorded by the handheld $\mathrm{ETCO}_{2} / \mathrm{SPO}_{2}$ monitor.

SV and GEF, as the indexes of myocardial function, were measured at baseline and hourly after resuscitation for a total of $4 \mathrm{hrs}$ with the PiCCO system. Serum concentration of cardiac troponin I (cTNI), which indicated the severity of cardiac injury, was measured at baseline and at $2 \mathrm{hrs}$, $4 \mathrm{hrs}$, and $24 \mathrm{hrs}$ after resuscitation with enzyme-linked immunosorbent assay (ELISA) kits (Meixuan Biotechnology Inc., Shanghai, China) according to the manufacturer's instructions. Aortic blood $\mathrm{pH}, \mathrm{PCO}_{2}, \mathrm{PO}_{2}$, hemoglobin, and lactate concentrations were measured at baseline and at $2 \mathrm{hrs}$ and $4 \mathrm{hrs}$ after resuscitation on $1.5 \mathrm{ml}$ of arterial blood samples with a Blood Gas/Electrolyte Analyzer (Model 5700, Instrumentation Laboratory, Lexington, MA).

Neurological function was evaluated according to the method of neurologic alertness score (NAS) developed by Tang et al. [22] at $24 \mathrm{hr}$ intervals for a total of $72 \mathrm{hrs}$. This quantitative NAS was based on the modifications of quantitative neurological deficit score [23]. The alertness score is acquired according to objective grading of level of consciousness, respiration, posture, water intake, eating, and self-care. The NAS was scored from 0 (coma) to 100 (fully alert). In earlier studies, the alertness score was highly correlated with the neurological deficit score $(r=-0.97)$ [24]. The NAS was examined and confirmed by two investigators who were blinded to the study. Serum concentrations of neuron-specific enolase (NSE) and S100B, considered as biochemical markers of cerebral injury, were measured at baseline and at $24 \mathrm{hrs}, 48 \mathrm{hrs}$, and $72 \mathrm{hrs}$ after resuscitation with ELISA kits (Meixuan Biotechnology Inc., Shanghai, China).

2.5. Statistical Analysis. Continuous variables were presented as mean \pm SD when data were normally distributed or as a median (25th, 75th percentiles) when data were not normally distributed. Normal distribution was confirmed with the Kolmogorov-Smirnov test. Variables were compared with one-way analysis of variance (ANOVA) or the Kruskal-Wallis test for nonparametric data. Comparisons between timebased measurements within each group were performed with repeated-measurement analysis of variance. If there was a significant difference in the overall comparison of groups, comparisons between any other two groups were made by the Bonferroni test. For the comparison of categorical variables such as ROSC, Fisher's exact test was used. A value of $P<0.05$ was considered significant.

\section{Results}

There were no significant differences in baseline hemodynamics, body temperature, arterial lactate, myocardial function, and serum biomarkers of heart and brain among the three groups (Tables 1, 4, and 5).

During CPR, the same trend of CPP was observed in all animals, in which the values were not significant among the three groups (Table 2). Six of the seven animals in each group were successfully resuscitated. No differences were observed in duration of CPR, epinephrine dosage, and number of shocks that were required for establishing ROSC and subsequent incidence of recurrent VF among the three groups (Table 3 ).

During $1.5 \mathrm{hrs}$ after resuscitation, body temperature was rapidly decreased from $37.1 \pm 0.5^{\circ} \mathrm{C}$ to $34.5 \pm 0.5^{\circ} \mathrm{C}$ in the combination group. Thereafter, a temperature of $32-34^{\circ} \mathrm{C}$ was maintained until $4 \mathrm{hrs}$ after resuscitation in animals that received hypothermia, followed by a rewarming rate of $1^{\circ} \mathrm{C} / \mathrm{h}$ for 4 hrs (Figure 1).

Following the observation of $4 \mathrm{hrs}$ after resuscitation, both of SV and GEF were significantly decreased in all animals when compared to the baseline values, which indicated significantly impaired myocardial function. However, postresuscitation myocardial function was gradually improved in all animals. The values of SV and GEF were significantly higher in the RIpostC alone and combination groups than those in the control group. Additionally, the values of SV and GEF were both greater in the combination group than the RIpostC alone group, in which the differences were significant among the two groups at $4 \mathrm{hrs}$ after resuscitation (Figures 2 and 3). cTNI kept increasing after resuscitation in all animals. However, slower increases in cTNI were observed in the RIpostC alone and combination groups, in which the values were significantly lower than the control group at 4 and $24 \mathrm{hrs}$ after resuscitation. $24 \mathrm{hr}$ cTNI was significantly lower in the combination group than the RIpostC group (Table 4). 
TABLE 1: Baseline characteristics.

\begin{tabular}{|c|c|c|c|}
\hline & RIpostC+TH $(n=7)$ & RIpostC $(n=7)$ & Control $(n=7)$ \\
\hline Body weight, $\mathrm{kg}$ & $37 \pm 2$ & $37 \pm 1$ & $38 \pm 2$ \\
\hline Heart rate, beats/min & $113 \pm 10$ & $109 \pm 7$ & $114 \pm 11$ \\
\hline Mean aortic pressure, $\mathrm{mm} \mathrm{Hg}$ & $110 \pm 4$ & $111 \pm 8$ & $112 \pm 6$ \\
\hline End-tidal $\mathrm{CO}_{2}, \mathrm{~mm} \mathrm{Hg}$ & $40 \pm 2$ & $39 \pm 2$ & $39 \pm 2$ \\
\hline Stroke volume, $\mathrm{ml}$ & $41 \pm 2$ & $41 \pm 2$ & $40 \pm 3$ \\
\hline Global ejection fraction, $\%$ & $31 \pm 3$ & $30 \pm 2$ & $30 \pm 2$ \\
\hline Body temperature, ${ }^{\circ} \mathrm{C}$ & $37.4 \pm 0.3$ & $37.4 \pm 0.5$ & $37.4 \pm 0.3$ \\
\hline
\end{tabular}

RIpostC, remote ischemic postconditioning; $\mathrm{TH}$, therapeutic hypothermia.

Data are presented as mean \pm SD.

TABLE 2: Coronary perfusion pressure during cardiopulmonary resuscitation.

\begin{tabular}{lccc}
\hline & RIpostC+TH & RIpostC & Control \\
\hline CPP in PC1, mm Hg & $20.1 \pm 2.7$ & $20.7 \pm 3.0$ & $21.3 \pm 3.1$ \\
CPP in PC3, mm Hg & $29.6 \pm 4.4$ & $29.3 \pm 4.3$ & $31.3 \pm 6.5$ \\
CPP in PC5, mm Hg & $27.4 \pm 2.6$ & $26.7 \pm 5.0$ & $28.0 \pm 7.0$ \\
\hline
\end{tabular}

RIpostC, remote ischemic postconditioning; TH, therapeutic hypothermia; CPP, coronary perfusion pressure; $\mathrm{PC} n$ indicates $n$ min after precordial compression.

Data are presented as mean \pm SD.

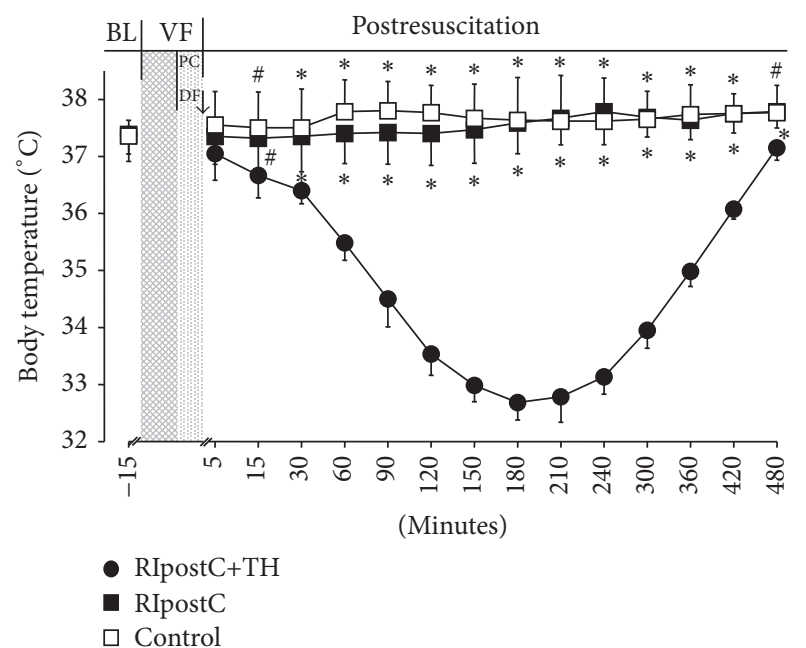

Figure 1: Body temperature, ${ }^{\circ} \mathrm{C}$. RIpostC, remote ischemic postconditioning; $\mathrm{TH}$, therapeutic hypothermia; $\mathrm{BL}$, baseline; VF, ventricular fibrillation; PC, precordial compression; DF, defibrillation. ${ }^{\#} P<0.05$ and ${ }^{*} P<0.01$ versus the RIpostC+TH group.

The same levels of arterial lactate were observed at baseline and after resuscitation in the RIpostC alone and combination groups, which returned to baseline levels in both groups at $4 \mathrm{hrs}$ after resuscitation, and were significantly lower when compared with the control group (Table 4).

The same duration of survival of $72 \mathrm{hrs}$ was achieved in all resuscitated animals. An even rate of survival was observed at $72 \mathrm{hrs}$ after resuscitation among the three groups. However, significantly higher NAS were observed in the RIpostC group than the control group. The combined RIpostC and $\mathrm{TH}$

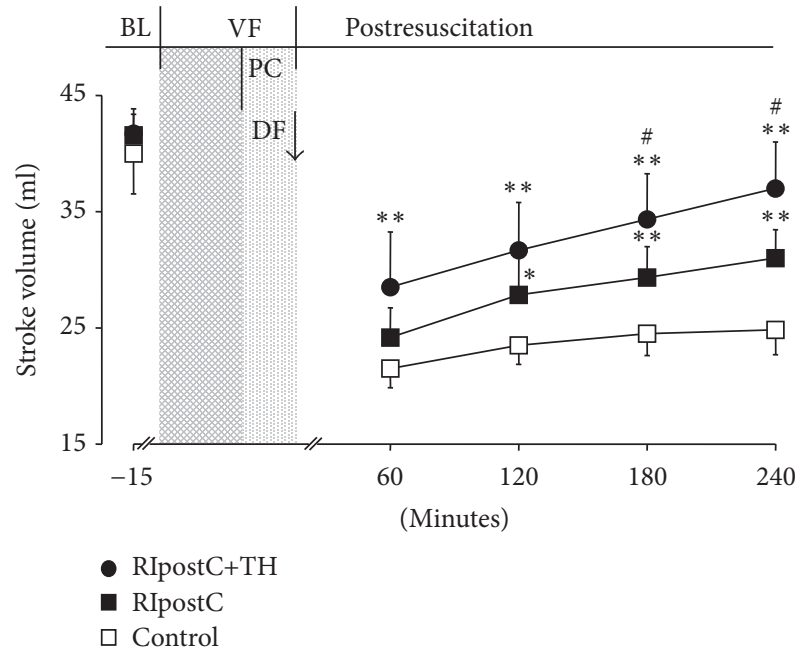

FIGURE 2: Stroke volume, ml. RIpostC, remote ischemic postconditioning; $\mathrm{TH}$, therapeutic hypothermia; $\mathrm{BL}$, baseline; VF, ventricular fibrillation; PC, precordial compression; DF, defibrillation. ${ }^{*} P<$ 0.05 and ${ }^{* *} P<0.01$ versus the control group; ${ }^{\#} P<0.05$ versus the RIpostC group.

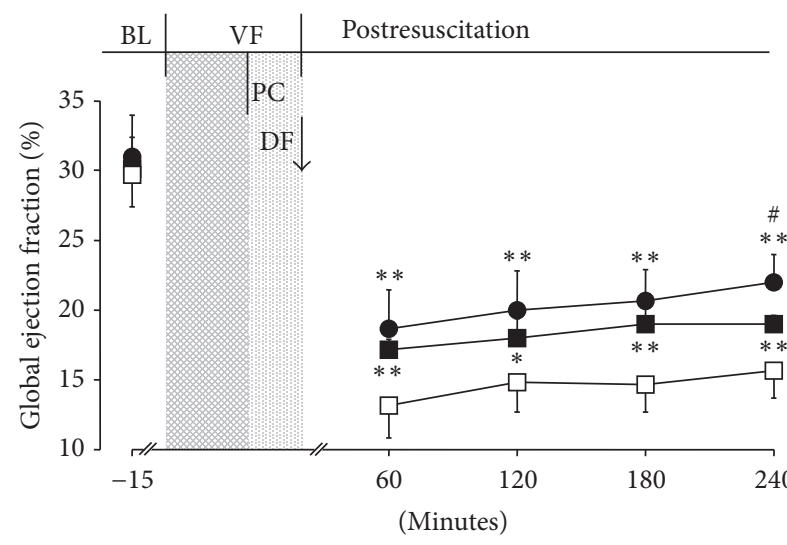

- $\mathrm{RIpostC}+\mathrm{TH}$

- RIpostC

$\square$ Control

Figure 3: Global ejection fraction, \%. RIpostC, remote ischemic postconditioning; $\mathrm{TH}$, therapeutic hypothermia; $\mathrm{BL}$, baseline; VF, ventricular fibrillation; PC, precordial compression; DF, defibrillation. ${ }^{*} P<0.05$ and ${ }^{* *} P<0.01$ versus the control group; ${ }^{\#} P<0.05$ versus the RIpostC group. 
TABLE 3: Cardiopulmonary resuscitation outcomes.

\begin{tabular}{lccc}
\hline & RIpostC+TH & RIpostC & Control \\
\hline ROSC & $6 / 7$ & $6 / 7$ & $6 / 7$ \\
Duration of CPR, min & $6.7 \pm 3.7$ & $7.0 \pm 3.7$ & $6.7 \pm 3.7$ \\
Number of shocks to ROSC & $2 \pm 2$ & $2 \pm 2$ & $2 \pm 2$ \\
Epinephrine dosage, mg & $1.3 \pm 1.2$ & $1.4 \pm 1.1$ & $1.3 \pm 1.1$ \\
Prevalence of recurrent VF & $1 \pm 2$ & $1 \pm 1$ & $1 \pm 2$ \\
\hline
\end{tabular}

RIpostC, remote ischemic postconditioning; TH, therapeutic hypothermia; ROSC, return of spontaneous circulation; CPR, cardiopulmonary resuscitation; VF, ventricular fibrillation.

Data are presented as mean $\pm \mathrm{SD}$.

TABLE 4: Serum cTNI and arterial lactate.

\begin{tabular}{|c|c|c|c|}
\hline & RIpostC+TH & RIpostC & Control \\
\hline \multicolumn{4}{|c|}{ cTNI, pg/ml } \\
\hline $\mathrm{BL}$ & $140 \pm 14$ & $140 \pm 16$ & $138 \pm 21$ \\
\hline PR $2 \mathrm{~h}$ & $150 \pm 8$ & $155 \pm 21$ & $159 \pm 26$ \\
\hline $\mathrm{PR} 4 \mathrm{~h}$ & $279 \pm 17^{* *}$ & $293 \pm 45^{*}$ & $361 \pm 53$ \\
\hline PR $24 \mathrm{~h}$ & $403 \pm 35^{* *, \#}$ & $453 \pm 30^{* *}$ & $523 \pm 43$ \\
\hline \multicolumn{4}{|c|}{ Arterial lactate, $\mathrm{mmol} / \mathrm{L}$} \\
\hline $\mathrm{BL}$ & $1.3 \pm 0.5$ & $1.5 \pm 0.4$ & $1.4 \pm 0.5$ \\
\hline PR $2 \mathrm{~h}$ & $2.6 \pm 0.4$ & $2.4 \pm 1.3$ & $5.3 \pm 3.7$ \\
\hline $\mathrm{PR} 4 \mathrm{~h}$ & $1.0 \pm 0.1^{*}$ & $1.2 \pm 0.7^{*}$ & $3.3 \pm 1.8$ \\
\hline
\end{tabular}

RIpostC, remote ischemic postconditioning; TH, therapeutic hypothermia; cTNI, cardiac troponin I; BL, baseline; PR, postresuscitation.

Data are presented as mean \pm SD.

${ }^{*} P<0.05$ and ${ }^{* *} P<0.01$ versus the control group; ${ }^{\#} P<0.05$ versus the RIpost $C$ group.

TABLE 5: Neurological function and cerebral injury.

\begin{tabular}{|c|c|c|c|}
\hline & RIpostC+TH & RIpostC & Control \\
\hline \multicolumn{4}{|c|}{ Neurologic alertness score } \\
\hline PR $24 \mathrm{~h}$ & $86 \pm 5^{* *, \#}$ & $76 \pm 9^{* *}$ & $49 \pm 9$ \\
\hline $\mathrm{PR} 48 \mathrm{~h}$ & $98 \pm 2^{* *, \#}$ & $88 \pm 8^{*}$ & $73 \pm 10$ \\
\hline PR $72 \mathrm{~h}$ & $99 \pm 1^{* *, \#}$ & $93 \pm 6^{*}$ & $86 \pm 4$ \\
\hline \multicolumn{4}{|c|}{ Neuron-specific enolase, $\mathrm{ng} / \mathrm{ml}$} \\
\hline $\mathrm{BL}$ & $8.6 \pm 3.0$ & $9.0 \pm 2.7$ & $9.0 \pm 2.2$ \\
\hline PR $24 \mathrm{~h}$ & $13.4 \pm 2.5$ & $15.4 \pm 2.3$ & $17.1 \pm 3.3$ \\
\hline $\mathrm{PR} 48 \mathrm{~h}$ & $19.3 \pm 1.5^{* *}$ & $20.9 \pm 1.9^{*}$ & $23.8 \pm 2.0$ \\
\hline PR $72 \mathrm{~h}$ & $18.5 \pm 1.4^{* *, \#}$ & $20.2 \pm 1.1^{* *}$ & $23.6 \pm 2.0$ \\
\hline \multicolumn{4}{|c|}{$\mathrm{S} 100 \mathrm{~B}, \mathrm{pg} / \mathrm{ml}$} \\
\hline $\mathrm{BL}$ & $696 \pm 30$ & $684 \pm 43$ & $692 \pm 41$ \\
\hline PR $24 \mathrm{~h}$ & $802 \pm 25$ & $818 \pm 63$ & $881 \pm 89$ \\
\hline PR $48 \mathrm{~h}$ & $988 \pm 92^{* *}$ & $1055 \pm 88^{*}$ & $1172 \pm 61$ \\
\hline PR $72 \mathrm{~h}$ & $886 \pm 33^{* *, \#}$ & $978 \pm 77^{*}$ & $1096 \pm 78$ \\
\hline
\end{tabular}

RIpostC, remote ischemic postconditioning; TH, therapeutic hypothermia; BL, baseline; PR, postresuscitation.

Data are presented as mean $\pm \mathrm{SD}$

${ }^{*} P<0.05$ and ${ }^{* *} P<0.01$ versus the control group; ${ }^{*} P<0.05$ versus the RIpostC group.

further improved postresuscitation neurological function, in which NAS was significantly higher than that in the RIpostC and control groups (Table 5). The levels of NSE and S100B were lower in animals that received RIpostC alone and combined with $\mathrm{TH}$, which were statistically significant at 48 and $72 \mathrm{hrs}$ after resuscitation when compared to the control group. $72 \mathrm{hr}$ NSE and S100B were significantly lower in the combination group than the RIpostC group (Table 5).

At necropsy, no significant abnormalities were observed on gross examination in all animals. 


\section{Discussion}

In the present study, we employed a clinically relevant, largeanimal model and demonstrated that remote ischemic conditioning via bilateral upper limbs was successfully induced at the stage of reperfusion during CA and resuscitation. Milder postresuscitation myocardial and neurological dysfunctions and reduced organ injuries of the heart and brain were achieved in animals that received RIpostC. In addition, the combined RIpostC and $\mathrm{TH}$ produced a greater effect of organ protection than the RIpostC alone and further improved postresuscitation cardiac and neurological outcomes.

During post-CA care, the degree of damage of heart and brain is closely related to the survival and quality of life of CA victims. New therapeutic strategies are needed to optimally mitigate the damage of these vital organs and therefore improve the prognosis of patients. RIpostC, serviced as ideal noninvasive and easily performed means, can be a feasible option. Previously, our group investigated the potential effectiveness of RIpostC in CA and resuscitation. A rat model was employed, and two limbs were chosen to implement RIpostC. Remote ischemic conditioning was finished by four cycles of 5 mins of limb ischemia and then 5 mins of reperfusion. RIpostC initiated during CPR or after successful resuscitation both significantly alleviated postresuscitation myocardial and cerebral dysfunctions [15]. In the present study, the same algorithm of remote stimulus was chosen to implement coincident with the beginning of CPR in swine. Improved myocardial and cerebral functions after resuscitation were also observed in animals that received RIpostC. Additionally, RIpostC mitigated postresuscitation cardiac and cerebral damage, as indicated by lower levels of serum biomarkers. These favourable outcomes might be attributed to two points: (1) During 5 mins of CPR, the ischemic period of RIpostC was induced by inflating the cuff around the bilateral upper limbs to stop their blood supply. Thus, a greater blood flow might be assigned to nearby vital organs such as the brain. (2) Considering that the severity of postresuscitation cardiac and cerebral dysfunction would get worse as the time went on, an earlier intervention such as RIpostC might prevent or slow down the process of cardiac and cerebral damage. Recently, a similar study demonstrated that RIpostC significantly reduced serum concentrations of biomarkers for cardiac damage but only slightly improved ventricular function and neurological recovery after resuscitation [25]. It might be due to a relatively shorter arrest of 8 mins and delayed RIpostC started 10 mins after resuscitation; the latter might weaken or lose the efficacy of organ protection. In a rat study of regional IR injury, the time window of intervention has occurred in the protective effects of RIpostC, in which cardiac protection was evident when applied 10 mins after reperfusion but disappeared when applied 30 mins after reperfusion [26].

Although cardiac and cerebral protection produced by RIpostC was manifested in our CA models, its potential mechanisms are unclear. Earlier investigations have confirmed that oxidant injury, inflammation, and cell apoptosis are all involved in the pathologic mechanisms of cardiac and cerebral injuries after successful CPR [27-30]. However, whether the protective effects of RIpostC are related to the alleviation of the above-mentioned pathological damage has not been explored. However, in diverse animal models of regional IR injury, RIpostC has been proven to provide cardiac and cerebral protection via the inhibition of oxidant injury, inflammation, and apoptosis. Li et al. [11] demonstrated that RIpostC significantly decreased myocardial infarction through the reduction in oxidant injury. Yu et al. [12] demonstrated that RIpostC protected the heart by modulating cell apoptosis through increasing the levels of $\mathrm{Bcl}-2 / \mathrm{Bax}$ and decreasing the levels of caspase-3. Another two studies demonstrated that RIpostC significantly attenuated brain injury; the neuroprotective mechanisms were potentially mediated by the reduction of oxidative stress and inflammatory response and also the anti-apoptotic action $[13,14]$.

In an attempt to enlarge the protective effects of vital organs after resuscitation, the effectiveness of combined RIpostC and TH was investigated. We chose a shorter duration of hypothermia of $4 \mathrm{hrs}$ based on our previous study [31]. Mild hypothermia was induced by surface cooling to aim at a temperature of $32-34^{\circ} \mathrm{C}$. Targeted temperature was quickly reached within $1.5 \mathrm{hrs}$ and then maintained until $4 \mathrm{hrs}$ after resuscitation. Synergistic protective effects were observed in the combination group, which resulted in greater improvements in postresuscitation myocardial and cerebral functions with lower levels of injury biomarkers when compared to the $\mathrm{RIpostC}$ alone. Considering that the protective efficacy of $\mathrm{TH}$ may be partly dependent on the duration of hypothermia, a longer duration of mild hypothermia may confer a stronger protective effect to the combination group [32].

Currently, no investigation has explored the interactive mechanisms of RIpostC and hypothermia. However, animal studies have confirmed that TH protected postresuscitation heart and brain by decreasing oxidant stress, inflammation, and apoptosis. Earlier, Ostadal et al. [33] demonstrated that HT produced cardiac protection through the suppression of oxidative stress following CA. Recently, another two studies demonstrated that $\mathrm{TH}$ protected post-CPR heart and brain by reducing the expression of inflammatory cytokines and cell apoptosis $[28,29]$. This similar pathological damage may be further alleviated by the combination of TH and RIpostC so as to produce greater protection of heart and brain after resuscitation. Future investigations are required to clarify the protective mechanisms of RIpostC and its interaction with TH in CA models.

There were some limitations in this study. First, we did not set the $\mathrm{TH}$ alone group. It is unknown whether better cardiac and neurological outcomes after CPR would be achieved by the combined RIpostC and TH than the TH alone. However, recent investigations demonstrated that under the condition of hypothermia remote ischemic conditioning still produced potent protection of vital organs $[34,35]$. Thus, RIpostC and TH might simultaneously reduce postresuscitation pathological damage so as to produce greater protection of heart and brain than the TH alone. Second, although we have observed postresuscitation cardiac and cerebral protection induced by RIpostC during this shorter observation period, we could not see the overall process of body recovery after CPR. A 
longer observation period would be better to fully evaluate the effectiveness of RIpostC in the future animal studies and clinical trials.

\section{Conclusion}

RIpostC was conducive to improving postresuscitation myocardial and cerebral functions and reducing their organ injuries in a porcine model of CPR. Its combination with $\mathrm{TH}$ further enhanced its protective effects.

\section{Competing Interests}

The authors do not have conflict of financial interests to disclose.

\section{Authors' Contributions}

Jiefeng Xu and Zeng Huang contributed equally to this study.

\section{Acknowledgments}

This study was funded by Zhejiang Provincial Medical Science Foundation (2013KYA190) and Ningbo's Municipal Natural Science Funds (2014A610251).

\section{References}

[1] G. Nichol, E. Thomas, C. W. Callaway et al., "Regional variation in out-of-hospital cardiac arrest incidence and outcome," The Journal of the American Medical Association, vol. 300, no. 12, pp. 1423-1431, 2008.

[2] C. Atwood, M. S. Eisenberg, J. Herlitz, and T. D. Rea, "Incidence of EMS-treated out-of-hospital cardiac arrest in Europe," Resuscitation, vol. 67, no. 1, pp. 75-80, 2005.

[3] W. Hua, L. F. Zhang, Y. F. Wu et al., "Incidence of sudden cardiac death in China: analysis of 4 regional populations," Journal of the American College of Cardiology, vol. 54, no. 12, pp. 1110-1118, 2009.

[4] J. P. Nolan, S. R. Laver, C. A. Welch, D. A. Harrison, V. Gupta, and K. Rowan, "Outcome following admission to UK intensive care units after cardiac arrest: a secondary analysis of the ICNARC Case Mix Programme Database," Anaesthesia, vol. 62, no. 12, pp. 1207-1216, 2007.

[5] C. Sasson, M. A. M. Rogers, J. Dahl, and A. L. Kellermann, "Predictors of survival from out-of-hospital cardiac arrest: a systematic review and meta-analysis," Circulation: Cardiovascular Quality and Outcomes, vol. 3, no. 1, pp. 63-81, 2010.

[6] C. Adrie, M. Adib-Conquy, I. Laurent et al., "Successful cardiopulmonary resuscitation after cardiac arrest as a 'sepsis-like' syndrome," Circulation, vol. 106, no. 5, pp. 562-568, 2002.

[7] R. W. Neumar, J. P. Nolan, C. Adrie et al., "Post-cardiac arrest syndrome: epidemiology, pathophysiology, treatment, and prognostication a consensus statement from the international liaison committee on resuscitation," Circulation, vol. 118, no. 23, pp. 2452-2483, 2008.

[8] I. Laurent, M. Monchi, J.-D. Chiche et al., "Reversible myocardial dysfunction in survivors of out-of-hospital cardiac arrest," Journal of the American College of Cardiology, vol. 40, no. 12, pp. 2110-2116, 2002.
[9] S. Laver, C. Farrow, D. Turner, and J. Nolan, "Mode of death after admission to an intensive care unit following cardiac arrest," Intensive Care Medicine, vol. 30, no. 11, pp. 2126-2128, 2004.

[10] R. O. Roine, S. Kajaste, and M. Kaste, "Neuropsychological sequelae of cardiac arrest," The Journal of the American Medical Association, vol. 269, no. 2, pp. 237-242, 1993.

[11] C.-M. Li, X.-H. Zhang, X.-J. Ma, and M. Luo, "Limb ischemic postconditioning protects myocardium from ischemiareperfusion injury," Scandinavian Cardiovascular Journal, vol. 40, no. 5, pp. 312-317, 2006.

[12] Y. Yu, X.-J. Jia, Q.-F. Zong et al., "Remote ischemic postconditioning protects the heart by upregulating ALDH2 expression levels through the PI3K/Akt signaling pathway," Molecular Medicine Reports, vol. 10, no. 1, pp. 536-542, 2014.

[13] P. Li, L. Su, X. Li et al., "Remote limb ischemic postconditioning protects mouse brain against cerebral ischemia/reperfusion injury via upregulating expression of Nrf2, HO-1 and NQO-1 in mice," International Journal of Neuroscience, vol. 126, no. 6, pp. 552-559, 2016.

[14] Q. Liu, S. N. Zhou, Y. D. Wang, F. Qi, Y. Song, and S. W. Long, "A feasible strategy for focal cerebral ischemiareperfusion injury: remote ischemic postconditioning," Neural Regeneration Research, vol. 9, no. 15, pp. 1460-1463, 2014.

[15] J. Xu, S. Sun, X. Lu, X. Hu, M. Yang, and W. Tang, "Remote ischemic pre- and postconditioning improve postresuscitation myocardial and cerebral function in a rat model of cardiac arrest and resuscitation," Critical Care Medicine, vol. 43, no. 1, pp. el2e18, 2015.

[16] M. Holzer, S. A. Bernard, S. Hachimi-Idrissi, R. O. Roine, F. Sterz, and M. Müllner, "Hypothermia for neuroprotection after cardiac arrest: systematic review and individual patient data meta-analysis," Critical Care Medicine, vol. 33, no. 2, pp. 414418, 2005.

[17] S. Ye, Y. Weng, S. Sun et al., "Comparison of the durations of mild therapeutic hypothermia on outcome after cardiopulmonary resuscitation in the rat," Circulation, vol. 125, no. 1, pp. 123-129, 2012.

[18] M.-S. Tsai, D. Barbut, H. Wang et al., "Intra-arrest rapid head cooling improves postresuscitation myocardial function in comparison with delayed postresuscitation surface cooling," Critical Care Medicine, vol. 36, no. 11 supplement, pp. S434S439, 2008.

[19] J. Guan, D. Barbut, H. Wang et al., "A comparison between head cooling begun during cardiopulmonary resuscitation and surface cooling after resuscitation in a pig model of cardiac arrest," Critical Care Medicine, vol. 36, no. 11, supplement, pp. S428-S433, 2008.

[20] S. Lin, D. C. Scales, P. Dorian et al., "Targeted temperature management processes and outcomes after out-of-hospital cardiac arrest: an observational cohort study," Critical Care Medicine, vol. 42, no. 12, pp. 2565-2574, 2014.

[21] J. Dankiewicz, S. Schmidbauer, N. Nielsen et al., "Safety, feasibility, and outcomes of induced hypothermia therapy following in-hospital cardiac arrest-evaluation of a large prospective registry," Critical Care Medicine, vol. 42, no. 12, pp. 2537-2545, 2014.

[22] W. Tang, M. H. Weil, S. Sun et al., "A comparison of biphasic and monophasic waveform defibrillation after prolonged ventricular fibrillation," Chest, vol. 120, no. 3, pp. 948-954, 2001. 
[23] R. A. Berg, C. W. Otto, K. B. Kern et al., "High-dose epinephrine results in greater early mortality after resuscitation from prolonged cardiac arrest in pigs: a prospective, randomized study," Critical Care Medicine, vol. 22, no. 2, pp. 282-290, 1994.

[24] W. Tang, M. H. Weil, R. B. Schock et al., "Phased chest and abdominal compression-decompression: a new option for cardiopulmonary resuscitation," Circulation, vol. 95, no. 5, pp. 1335-1340, 1997.

[25] M. Albrecht, P. Meybohm, O. Broch et al., "Evaluation of remote ischaemic post-conditioning in a pig model of cardiac arrest: a pilot study," Resuscitation, vol. 93, pp. 89-95, 2015.

[26] M. Basalay, V. Barsukevich, S. Mastitskaya et al., "Remote ischaemic pre- and delayed postconditioning-similar degree of cardioprotection but distinct mechanisms," Experimental Physiology, vol. 97, no. 8, pp. 908-917, 2012.

[27] F. S. Hackenhaar, F. Fumagalli, G. Li Volti et al., "Relationship between post-cardiac arrest myocardial oxidative stress and myocardial dysfunction in the rat," Journal of Biomedical Science, vol. 21, article 70, 2014.

[28] P. Meybohm, M. Gruenewald, M. Albrecht et al., "Hypothermia and postconditioning after cardiopulmonary resuscitation reduce cardiac dysfunction by modulating inflammation, apoptosis and remodeling," PLoS ONE, vol. 4, no. 10, Article ID e7588, 2009.

[29] P. Meybohm, M. Gruenewald, K. D. Zacharowski et al., "Mild hypothermia alone or in combination with anesthetic postconditioning reduces expression of inflammatory cytokines in the cerebral cortex of pigs after cardiopulmonary resuscitation," Critical Care, vol. 14, no. 1, article no. R21, 2010.

[30] X. Xie, J. Zhang, D. Chen et al., "Effects of ghrelin on postresuscitation brain injury in a rat model of cardiac arrest," Shock, vol. 43, no. 5, pp. 490-496, 2015.

[31] Y. Li, G. Ristagno, J. Guan et al., "Preserved heart rate variability during therapeutic hypothermia correlated to $96 \mathrm{hrs}$ neurological outcomes and survival in a pig model of cardiac arrest," Critical Care Medicine, vol. 40, no. 2, pp. 580-586, 2012.

[32] G. J. Suh, W. Y. Kwon, K. S. Kim et al., "Prolonged therapeutic hypothermia is more effective in attenuating brain apoptosis in a swine cardiac arrest model," Critical Care Medicine, vol. 42, no. 2, pp. e132-e142, 2014.

[33] P. Ostadal, M. Mlcek, A. Kruger et al., "Mild therapeutic hypothermia is superior to controlled normothermia for the maintenance of blood pressure and cerebral oxygenation, prevention of organ damage and suppression of oxidative stress after cardiac arrest in a porcine model," Journal of Translational Medicine, vol. 11, article no. 124, 2013.

[34] H. A. Jensen, S. Loukogeorgakis, F. Yannopoulos et al., "Remote ischemic preconditioning protects the brain against injury after hypothermic circulatory arrest," Circulation, vol. 123, no. 7, pp. 714-721, 2011.

[35] Z. Y. Wu, Z. W. Wang, R. Hu, Z. Zhou, L. C. Li, and Z. F. Mao, "Role of Nrf2 signal pathway in rats with deep hypothermia ischemia/reperfusion injury undergoing remote postconditioning," Genetics and Molecular Research, vol. 14, no. 1, pp. 492-499, 2015. 


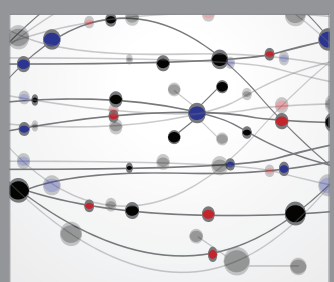

The Scientific World Journal
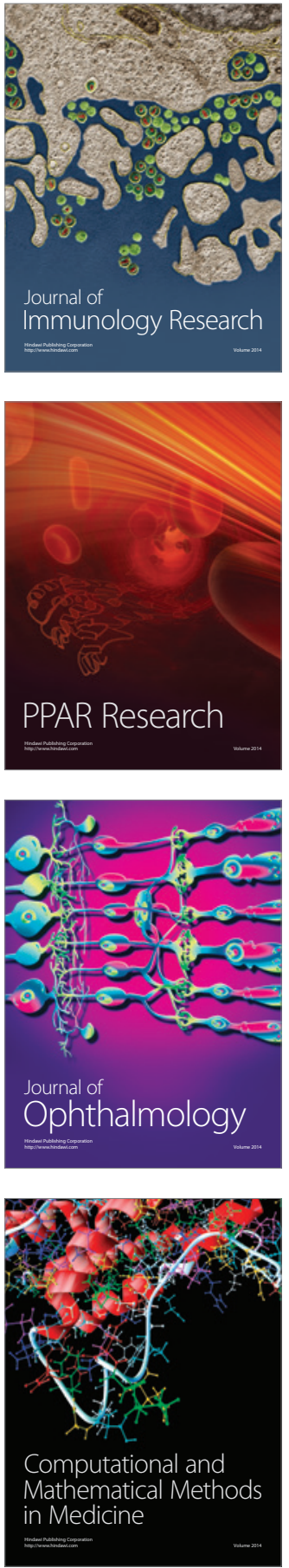

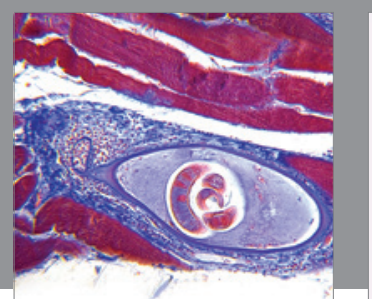

Gastroenterology Research and Practice

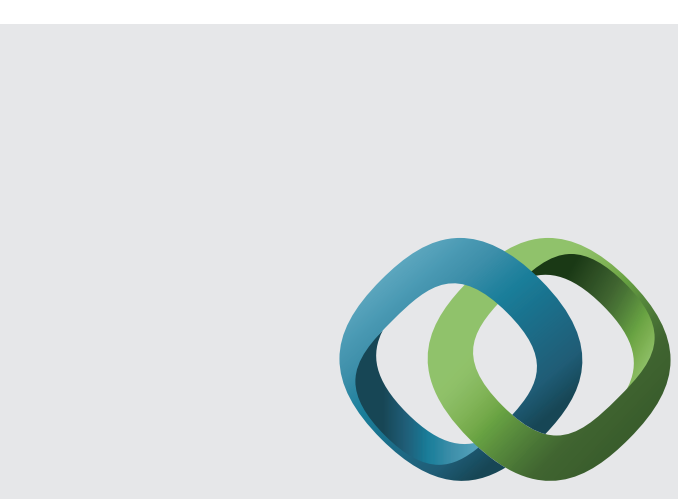

\section{Hindawi}

Submit your manuscripts at

http://www.hindawi.com
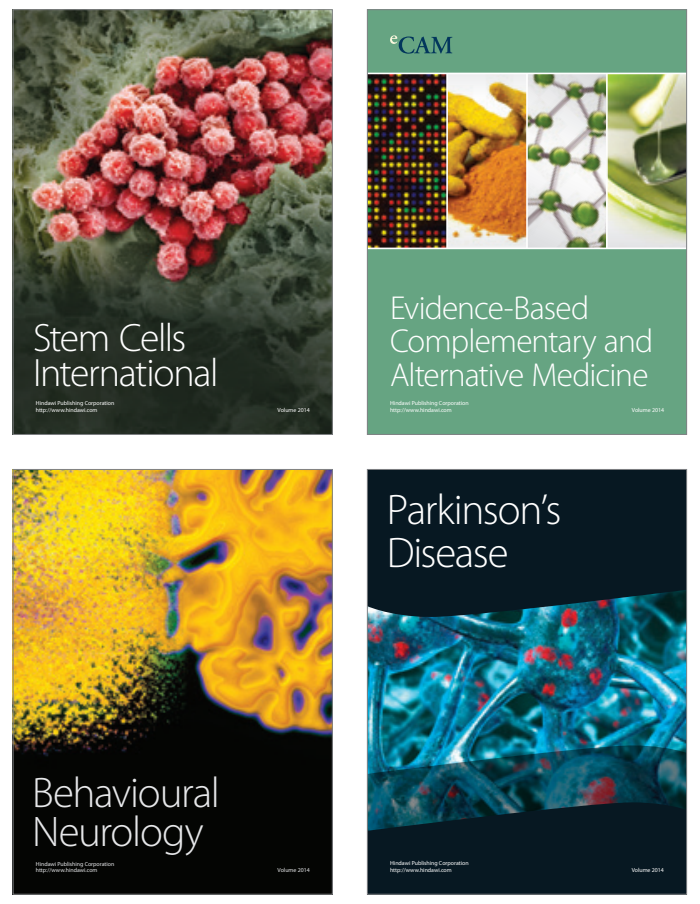
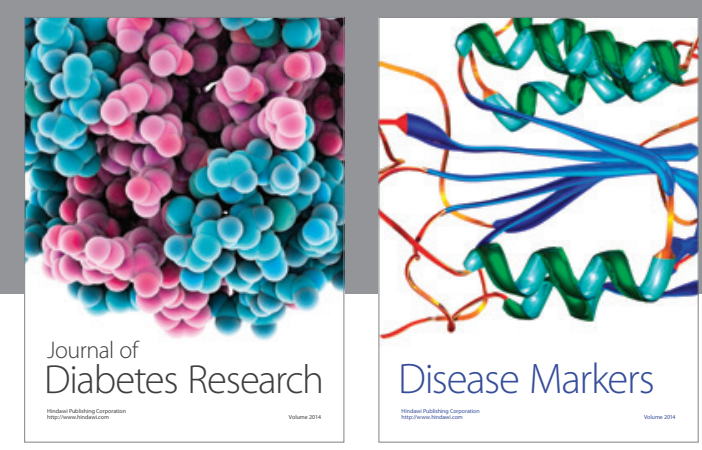

Disease Markers
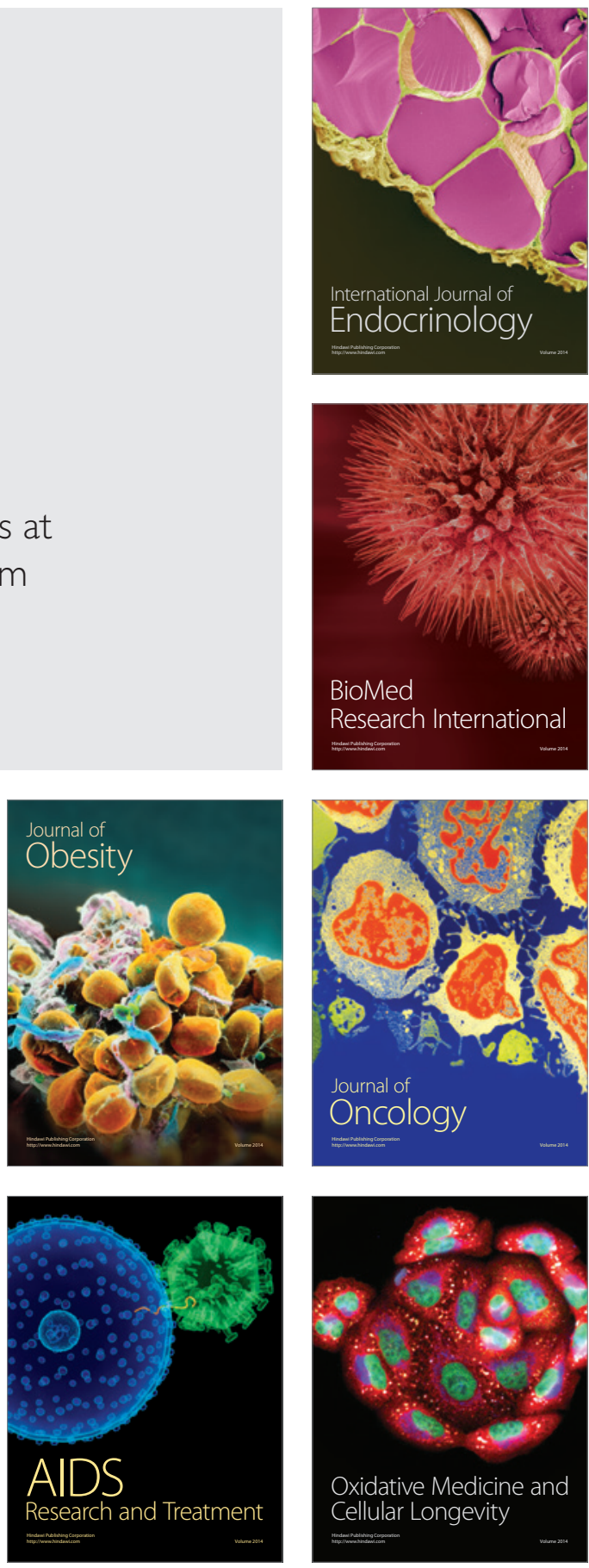